Review Article

\title{
Disease X: Exploring the Unexplored, Knowable Unknown
}

\author{
Rina Tilak', Sajal Bhattacharya ${ }^{2}$, VW Tilak $^{3}$, Shakya Sinha ${ }^{4}$
}

${ }^{1}$ Scientist ' $G$ ', ${ }^{3}$ Ex Dean, Armed Forces Medical College, Pune, India.

${ }^{2}$ Professor (Associate), ${ }^{4}$ Research Scholar, Postgraduate Department of Zoology, Asutosh College (University of Calcutta), Kolkata, West Bengal, India.

DOI: https://doi.org/10.24321/0019.5138.202183

$\begin{array}{llll}\text { I } & \mathbf{N} & \mathbf{F} & \mathbf{O}\end{array}$

\section{Corresponding Author:}

Shakya Sinha, Postgraduate Department of Zoology, Asutosh College (University of Calcutta), West Bengal, India.

E-mail Id:

shakyasinha24@gmail.com

Orcid Id:

https://orcid.org/0000-0003-4758-1693

How to cite this article:

Tilak R, Bhattacharya S, Tilak VW, Sinha S. Disease $X$ : Exploring the Unexplored, Knowable Unknown. J Commun Dis. 2021;53(4):135-139.

Date of Submission: 2021-12-02

Date of Acceptance: 2021-12-23

\section{$\begin{array}{llllllll}\mathbf{A} & \mathbf{B} & \mathbf{S} & \mathbf{T} & \mathbf{R} & \mathbf{A} & \mathbf{C} & \mathbf{T}\end{array}$}

The glaring uncertainty regarding the identity of certain pathogenic bio-agents having potential for a severe global public health security threat has spurned the international health agencies to classify it as Disease X, which has been added to the "blueprint priority diseases" as a placeholder name for a "knowable unknown" pathogen by the WHO. The zoonotic diseases of viral origin and the synthetic viruses are acknowledged as the most likely agents of Disease $X$ causing public health emergencies of international concern in times to come. The challenges in the rapid containment of Disease $X$ pandemics could be the inability for early detection of the infectious agents, presence of a large immunologically naïve population, lack of knowledge of clinical spectrum, no pathogen-specific effective drugs and/ or vaccines and sudden surge of patients requiring hospitalisation which collectively compromise the public health system especially in resource stricken countries and leads to its eventual collapse. Well-equipped field laboratories, sentinel surveillance centres for viruses and other microbial pathogens are needed in strategically important and sensitive areas of concern for monitoring for early detection of possible bio-agents of Disease $X$, if any. In a changing geo-socio political scenario, use of the synthetic viruses as a potential bio-weapon for mass destruction and/ or for the economic breakdown of a targeted country should not be ruled out. Thus, capacity building, global technical collaboration and stringent international regulations to prevent the development of bioweapons are also obligatory to prevent any possible future pandemics of Disease X.

Keywords: Disease X, Pandemics, Surveillance, Bio-weapons, Blueprint Priority Diseases

\section{Introduction}

The world today is more crowded, more polluted, less stable ecologically but more vulnerable to diseases and disruptions. ${ }^{1}$ The world is perched on a ticking time bomb holding a vast number of undiscovered, potentially deadly disease-causing pathogens that await opportunities to unleash unprecedented havoc at the slightest provocation by man. With each passing day, the world waits with bated breath the reporting of the first patient of the dreaded 'Disease $X$ ', an insidious unknown threat. 
It is not surprising therefore that WHO in February 2018, added Disease $X$ to the shortlist of the "blueprint priority diseases" as a placeholder name for a "knowable unknown" pathogen. ${ }^{2-4}$ 'Disease $X$ ' currently symbolises the awareness that a pandemic could be caused by an unknown, hypothetical pathogen that is currently not known to cause human infection or disease. ${ }^{5}$ The potential of an unknown pathogen - 'Disease $X$ ', to cause epidemics or pandemics in future has ensured its inclusion by the WHO in the list of priority diseases along with COVID-19, Crimean-Congo Haemorrhagic Fever (CCHF), Ebola virus disease and Marburg virus disease, Lassa fever, Middle East Respiratory Syndrome Coronavirus (MERS-CoV) and Severe Acute Respiratory Syndrome (SARS), Nipah and henipaviral diseases, Rift Valley fever and Zika to enable planning and building capabilities to address such an event anytime in the future. ${ }^{2,3}$ This list of priority diseases is meticulously updated by the WHO every year to accommodate any new perceived emerging threat.

The scientific fraternity, as of date, is actively engaged in a spirited debate whether COVID-19, caused by the SARSCoV-2, can truly be considered to be the first Disease $X$, as it meets all the criteria to be termed so. ${ }^{6}$ The impact of the diseases like Ebola, SARS, MERS, Nipah, Zika etc. in the recent past has tested man's perseverance and aptitude to overcome the stupendous morbidity and mortality caused by these diseases. The time is not up; there are presumably a large number of diseases, especially zoonotic, which are poised to spill over to man and thus pose fresh public health challenges of gargantuan magnitude. Seeing the current trend of the pandemic, it is widely speculated that the next pandemic would be far more impactful and may traverse all limits of human imagination. The era of pandemics has just begun; the impact is beginning to be felt. This review is an attempt to explore the unknown pathogens - Disease $X$, hitherto undetected in order to assess and analyse their epidemic and pandemic potential, if any. This study would be helpful to formulate the policies and programmes, early preparedness and strategies to prevent or contain the future outbreak of such diseases, if it happens.

\section{Causative Agents of Disease $X$}

\section{Viruses}

Viruses, undeniably, form a major group of organisms that are likely to cause future pandemics of Disease $X$, a fact clearly evidenced from the list of recent pandemics, all of which have viral aetiology and the same is also visibly reflected in the list of priority diseases of WHO which need to be kept under close watch.

It is currently estimated that about 1.67 million viruses are still unknown to mankind with about 631,000 to 827,000 viruses with a likely capability to infect humans. ${ }^{7}$ However, as of date, we can boast of knowing over 200 viruses with infectivity potential to humans. ${ }^{8}$ Therefore, majority of viruses, i.e. more than $99 \%$ are still unknown and are speculated to possess the innate ability to not only infect man but also initiate pandemic situations. It is thus a matter of intense scientific speculation and debate that from this large repertoire of unknown viruses, how many can actually emerge as the next Ebola or COVID-19?

To address this fatalistic question, it is necessary to explore this vast ocean of unknown viruses. In particular, RNA viruses are of special significance as they are the causative agent for over $94 \%$ of zoonotic viral diseases, possibly owing to their wide host range, high mutability and high adaptability. ${ }^{9}$ To be forewarned is to be forearmed, and we under no circumstances can afford to indulge in the redundant luxury of complacency at this juncture.

\section{Bacteria}

The bacterial infections cannot be overlooked as agents of the next Disease $X$ pandemic. Recent studies by a group of scientists revealed that bacterial diseases constitute about $54.3 \%$ of the emerging infectious diseases. ${ }^{10}$ According to recent evidence, there are possibly 0.8 to 1.6 million prokaryotic organisms, and most of them are yet to be discovered. ${ }^{11}$ Human exposure to these potential pathogens may push us towards the next epidemic or pandemic. Adding to that, increasing incidences of antibiotic resistance to the most advanced antibiotics - Carbapenems (a class of antibiotics used for known or suspected multidrug-resistant (MDR) bacterial infections) and Colistin (an antibiotic used as a last-resort in multidrug-resistant gram-negative infections as well as pneumonia) is touted to usher in an era of highly resistant bacteria. Some cases in point are antibiotic resistance in gonorrhoea, tuberculosis, salmonellosis. ${ }^{12}$ It's prudent that the widespread and indiscriminate use of antibiotics be timely redressed to prevent an eventuality of Disease $X$ in the future.

\section{Synthetic Viruses}

Genome sequencing and oligonucleotide synthesis are revolutionary technologies through which synthetic genomes can be constructed, be it the RNA or DNA viruses. ${ }^{13}$ Possibilities of genome-based interference of an already dynamic gene pool of known and unknown viruses is a concerning avenue for future evaluation. ${ }^{14}$ Technically RNA viruses are relatively easier to synthesise, given their small genome size and the availability of reverse transcription technology. ${ }^{15}$ Poliovirus and $\phi X 174$ bacteriophage are two examples of the first synthetic infectious viruses made sans any natural template. The potential to create such synthetic viruses is decidedly available with many research laboratories globally, which are known to be equipped with the skill and competence to undertake 
such research. However, the choice of whether to develop such viruses for gene therapy or as bioweapons is solely at the discretion of man and his intent. The impact of these synthetic viruses, whether released accidentally or intentionally could be simply disastrous as the populations would be highly susceptible due to no prior exposure. It is thus highly pertinent that stringent regulations governing such research facilities be put in place to prevent the development of bioweapons and their accidental release which might initiate future pandemics of Disease $X$.

\section{Mode of Transmission}

Historically most of the epidemics and pandemics that mankind has faced till now were air-borne or vectorborne or contagious. Similarly, the most likely modes of transmission of these probable infectious agents of Disease $X$ could either be air/ aerosol borne or vector-mediated or by direct contact. Air/ aerosol borne agents and contagious diseases have the capability to infect far more numbers in the shortest time span as compared to vector-borne pathogens, which is greatly facilitated by the increased air travel due to trade and tourism. Rapid globalisation has aided a faster pace of the spread of these infections from one part of the globe to another. It is thus significant and heartening that the recognition of the entity 'Disease $X$ ' and its potential to cause pandemics has advantageously ensured focused research in developing capabilities for faster identification of the infectious agent and tools to contain its spread in its place of origin, thereby buying time for the development of treatment modalities or vaccines in other unaffected countries. The vector-mediated diseases as a cause of Disease $X$ pandemics is also a distinct probability and cannot be ignored. Global warming, changing climatic conditions, rapid urbanisation, global travel and trade etc. have all favoured establishments of vectors in newer areas and also enhanced exposure of humans to the vectors. The emergence of newer vectors and reservoirs of vectorborne diseases is itself challenging. It is thus pertinent that we remember this mode of transmission as well when we deliberate on the possible routes of transmission of infectious agents likely to cause Disease $X$.

\section{Factors Favouring Disease $X$ Outbreaks Man - The Culprit}

The focus on "One health concept" could not have been better timed than now. It is imperative to remind ourselves that the delicate yet dynamic interaction amongst humans, wildlife and livestock has been severely impacted to meet the demands of the growing human population, especially in the urban and peri-urban areas. This situation facilitates the man-animal contact and conflict. Humans have encroached upon the forests, traded wildlife, brought about landuse changes, altered agricultural practices, facilitated rapid urbanisation, global trade and movement coupled with climate change, which have all resulted in enhanced exposure of man to wild animals, especially bats and other animal reservoirs of diseases. Bats and pigs are well known as rich reservoirs of zoonotic infections and as 'mixing pots' for multiple viruses which then produce new recombinant viruses that tend to spill over to humans because of their close proximity to them. ${ }^{16,17} \mathrm{~A}$ pandemic mediated by these recombinants definitely qualifies to be ranked as one of the reasons for a likely outbreak of Disease $X$.

\section{Climate Change}

Climate change is one of the defining challenges of our time owing to its wider implications on biodiversity, especially on humans, and various life-supporting systems ${ }^{18}$ be it in the form of global warming affecting the daily life of humans or its varied implications including unleashing newer diseases, the emergence of diseases in newer areas or facilitating zoonotic spillover. ${ }^{19}$ The melting of polar ice caps due to global warming may release certain ancient viruses or their genetic materials which have been buried in the ice for thousands of years and were dormant in icecold temperatures. Whether these long-buried viruses after being exposed could retain their potential to infect the suitable host cells, if found, and could be a threat to biodiversity including humans, is a matter of conjecture. ${ }^{18}$

\section{Emergence of Recombinants in the Reservoir and Zoonosis}

Zoonotic virulent RNA viruses have been a matter of concern for the scientific fraternity since long due to their high mutability and varied diversity in animal reservoirs. The role of multiple infections in the zoonotic reservoirs and the possibility of a resultant recombinant, which then can get transmitted to humans by vectors, are likely to throw deadly surprises for which we are regrettably unprepared. It is likely that the diseases owing to such recombinants may present with atypical clinical symptoms that may go unnoticed to start with. If such diseases are also blessed with the capability of being aerosol transmitted, the situation may turn out to be highly explosive.

\section{The Way Forward}

The war against Disease $X$ is launched; it's 'THE' time to upgrade our arsenal and capabilities to tackle the unseen enemy. The efforts need to be focused on the development of capabilities for identification and genome sequencing of the remaining list of unknown viruses, quick identification of unknown pathogens causing outbreaks, capacity building for development of treatment strategies and vaccines within a short time frame, improvisation of regulations to permit the same, establishment of sentinel surveillance centres in all regions focused on monitoring zoonotic reservoirs for newer infections, recombinant viruses, and vector 
species. It's time that all the countries are brought on board by setting them up on the same scientific and technical platform so that effective global surveillance systems may be established and preventive and treatment technologies can be developed to collectively fight the unseen enemy in the most efficient manner. Unity in efforts to prevent the Disease X pandemic is the 'keyword' as of now and a reminder that united we stand and divided we fall.

\section{Discussion}

Man has been an incessant victim of Nature's fury, be it the innumerable diseases that have plagued mankind since time immemorial or the catastrophe heaved by the multiple natural calamities which nearly ravaged mankind in impacted areas. The onslaughts of new disease entities in the last decade have challenged mankind in an unprecedented manner. Prediction models have identified Emerging Infectious Disease (EID) hotspots in Africa, Latin America and South Asia with countries like China, India and Bangladesh being the brightest, thus indicating the highest risk. These countries, besides being densely populated, also have heavily forested areas. With rapid urbanisation, this forest cover is slowly, yet steadily declining thereby increasing the risk of enhanced contact between man and wild animals. ${ }^{20}$ The scientific literature is a brutal reminder to man to curb his territorial ambitions to prevent increased opportunities of man-wildlife interactions especially in highly biodiversity-rich areas of the world, as zoonosis is the reason behind about $75 \%$ of the EID's in recent times. ${ }^{20,21}$

The current pandemic, COVID-19, has been a boon in a way that it has jolted us out of the blissfully ignorant state to an alert and anticipatory state, which will ensure diversion of appropriate scientific resources and technology to enable better preparedness to deal with this unknown enemy in a decisive and informed manner. It's vital that we understand and identify the areas from where the likelihood of origin of Disease ' $X$ ' is more, so that regional strategies and policies may be efficiently streamlined for its prevention and mitigation to prevent its wider spread and a pandemic situation. Such areas would invariably have the common characteristic of having high wildlife diversity (especially bats and other wild mammal species) coupled with high human interaction with these reservoirs as well. The changing dynamics of pathogens, especially viruses, could become a challenge for the scientific fraternity trying to develop preventive strategies for certain Disease X. Shift of the Dengue virus towards the 'lymphotropism' from their 'neurotropic' ancestors is a well-known instance. ${ }^{22}$ Apprehensions have been raised by investigators that this kind of shift in the disease dynamics could also be seen for other pathogens including the recent pandemic causing SARS-CoV-2. ${ }^{14}$ Hence, dedicated research, surveillance and monitoring are an imperative necessity to know the unknown pathogens and preventive strategies are required to prevent or contain the emergence of such pathogens and outbreaks if any.

Co-evolution of microbes/ pathogens with their hosts followed by sustainable ecosystem functioning are the basis of cooperative co-existence of different forms of life and ecological homeostasis on this earth since time immemorial. Deforestation, land-use change, global warming, globalisation, global trade, and travel have a profound impact and are found to be important drivers on the emergence, reemergence and spread of infectious diseases. Therefore, sustainable developmental policies with reference to long term consequences on human health ${ }^{23}$ and ecological equilibrium should be adopted and regarded as a management tool to minimise the chances of pathogen spillover from the wild environment. The global health authorities are now in agreement with adopting the 'One health' policy in order to make the world a safer place by attempting to restrict the unknown zoonotic pathogens in the wild environment and thus prevent their possible spillover to humans. ${ }^{21}$ Comprehensive environmental health management policies under the 'one health' concept following the principle of conservation medicine encompassing humans, flora and fauna, and environment as a whole could be an effective management strategy in preventing and/ or reducing the threat of emergence of $X$ pathogen as well as the reemergence of endemic diseases from wild reservoir hosts. ${ }^{16}$ In order to achieve the objectives discussed above, sustained virus and other microbial research of medical and veterinary importance along with the establishment of a diagnostic laboratory in both urban and rural setups are immensely important and essential.

\section{Conclusion}

It is most opportune and apt that Disease $\mathrm{X}$ has been listed in the "blueprint priority diseases" by the WHO. This will ensure that countries all over the world appreciate the likelihood of Disease $X$ pandemic and also acknowledge the importance of advance planning for prevention against diseases originating from yet unidentified sources, so that we are not caught napping. The stage is set for the researchers to rise to the challenge of unravelling the mysterious world of unknown viruses/ other pathogens and deciphering their pandemic potential. Efforts have to be channelized to lessen the time lag between the identification of the cause of outbreaks and the obligatory sanctions for the development of vaccines/treatments, in order to thwart the progression of outbreaks into a "public health emergency".

\section{Source of Funding: None \\ Conflict of Interest: None}




\section{References}

1. Bhattacharya S. Mosquito borne diseases in India with special reference to malaria vectors and their control. J Asiatic Soc. 2009;L1(2):15-34.

2. World Health Organization [Internet]. 2018 Annual review of diseases prioritized under the Research and Development Blueprint. Geneva, Switzerland; 2018 [cited 2021 Nov 30]. Available from: https://www. who.int/docs/default-source/blue-print/2018-annualreview-of-diseases-prioritized-under-the-research-anddevelopment-blueprint.pdf?sfvrsn=4c22e36_2

3. World Health Organization [Internet]. Prioritizing diseases for research and development in emergency contexts; 2021 [cited 2021 Nov 30]. Available from: https://www.who.int/activities/prioritizing-diseasesfor-research-and-development-in-emergency-contexts

4. Friedrich MJ. WHO's blueprint list of priority diseases. JAMA. 2018 May;319(19):1973. [PubMed] [Google Scholar]

5. Simpson S, Kaufmann MC, Glozman V, Chakrabarti A. Disease $X$ : Accelerating the development of medical countermeasures for the next pandemic. Lancet Infect Dis. 2020 May;20(5):e108-15. [PubMed] [Google Scholar]

6. Jiang S, Shi ZL. The first disease $X$ is caused by a highly transmissible acute respiratory syndrome coronavirus. Virol Sin. 2020 Jun;35(3):263-5. [PubMed] [Google Scholar]

7. Carroll D, Daszak P, Wolfe ND, Gao GF, Morel CM, Morzaria S, Pablos-Méndez A, Tomori O, Mazet JAK. The global virome project. Science. 2018;359(6378):8724. [PubMed] [Google Scholar]

8. Woolhouse M, Scott F, Hudson Z, Howey R, Chase-Topping M. Human viruses: discovery and emergence. Philos Trans R Soc Lond B Biol Sci. 2012 Oct;367(1604):2864-71. [PubMed] [Google Scholar]

9. Woolhouse ME, Adair K, Brierley L. RNA viruses: a case study of the biology of emerging infectious diseases. Microbiol Spectr. 2013 Oct;1(1):10. [PubMed] [Google Scholar]

10. Jones KE, Patel NG, Levy MA, Storeygard A, Balk D, Gittleman JL, Daszak P. Global trends in emerging infectious diseases. Nature. 2008 Feb;451:990-3. [PubMed] [Google Scholar]

11. Louca S, Mazel F, Doebeli M, Parfrey LW. A census-based estimate of Earth's bacterial and archaeal diversity. PLoS Biol. 2019 Feb;17(2):e3000106. [PubMed] [Google Scholar]

12. World Health Organization [Internet]. Antibiotic resistance; 2020 [cited 2021 Nov 30]. Available from: https://www.who.int/news-room/fact-sheets/ detail/antibiotic-resistance\#: : :text=Antibiotic\%20 resistance\%20occurs\%20when\%20bacteria,caused\%20
by\%20non\%2Dresistant $\% 20$ bacteria

13. Heather JM, Chain B. The sequence of sequencers: The history of sequencing DNA. Genomics. 2017 Jan;107(1):1-8. [PubMed] [Google Scholar]

14. Chatterjee R, Bhattacharya S. Could novel corona virus (SARS-CoV-2) be the evolving face of a new generation of genetically complex epidemiological challenges? Malaysian J Med Res. 2020;4(2):49-52. [Google Scholar]

15. Stauft CB, Wimmer E. Synthetic viruses. John Wiley \& Sons, Ltd; 2019;1-7.

16. Bhattacharya S, Sinha S, Tilak R, Mardihusodo SW. The relationship between bats and human coronavirus: an exploratory review. J Health Soc Sci. 2020;5(2):219-30. [Google Scholar]

17. Tilak R, Bhattacharya S, Sinha S. Genotype 4 reassortant Eurasian avian-like H1N1 swine flu virus: an emerging public health challenge. Asian Pac J Trop Med. 2021;14(3):97-8. [Google Scholar]

18. Bhattacharya S. Challenges of mosquito-borne diseases in the changing ecological conditions. J Asia Soc. 2011;LIII(3):109-18.

19. Bhattacharya S, Sinha S, Baidya D, Tilak R. Emergence of a zoonotic pathogen - novel Coronavirus (SARS-CoV-2) in the context of changing environment. J Commun Dis. 2020;52(2):18-24. [Google Scholar]

20. Allen T, Murray KA, Zambrana-Torrelio C, Morse SS, Rondinini C, Di Marco M, Breit N, Olival KJ, Daszak. Global hotspots and correlates of emerging zoonotic diseases. Nat Commun. 2017 Oct;8(1):1124. [PubMed] [Google Scholar]

21. World Health Organization [Internet]. WHO DirectorGeneral's remarks at the 1st virtual meeting of the One Health High Level Expert Panel - 17 May 2021; [cited 2021 Nov 30]. Available from: https://www.who. int/director-general/speeches/detail/who-directorgeneral-s-remarks-at-the-1st-virtual-meeting-of-theone-health-high-level-expert-panel-17-may-2021

22. Monath TP. Dengue: the risk to developed and developing countries. Proc Natl Acad Sci U S A. 1994 Mar;91(7):2395-400. [PubMed] [Google Scholar]

23. Jones KE, Patel NG, Levy MA, Storeygard A, Balk D, Gittleman JL, Daszak P. Global trends in emerging infectious diseases. Nature. 2008;451:990-3. [PubMed] [Google Scholar] 